\title{
MODELING OF THE DISPERSION MAGNETIC FIELDS FOR THE SINGLE-PHASE TRANSFORMERS
}

\author{
V. SOLCANU ${ }^{1}$, M. GAICEANU ${ }^{1}$, M. CONSTANTINESCU ${ }^{2}$, GHE. SAMOILESCU ${ }^{2}$, A. SOTIR ${ }^{2}$ \\ ${ }^{1}$ Dunarea de Jos University of Galati, 800008, Romania, ${ }^{2}$ Naval Academy "Mircea cel Batran", Constanta, Romania \\ E-mail: vasilesolcanu@dedeman.ro,marian.gaiceanu@ugal.ro,gheorghe.samoilescu@anmb.ro
}

\begin{abstract}
Different methods of dispersion magnetic fields solutions for single phase transformers are presented in this paper. The transformers are with cylinder windings placed onto a single column. The transformer construction transformer is in the mantle. The analytic equation expression of finding dispersion magnetic fields have been delivered and the obtained solution has been verified by the experimental field measurements.
\end{abstract}

Keywords: dispersion leakage magnetic field of /, single phase transformer, coil, electromagnetic compatibility (EMC), electromagnetic interference (EMI), core coils.

\section{INTRODUCTION}

One of the basic concerns in electromagnetic compatibility is to plan, specify and design electrical and electronic systems so that they can be installed, in their operational environment, without creating or being susceptible to interference [1], [2]. In this regard, attention must be paid to a number of important factors that influence electromagnetic compatibility. In particular, major sources of electromagnetic interference, coupling modes and susceptibility points or conditions must be considered.

Thus, since the system definition phase, the designer should foresee and analyze the electromagnetic interference (EMI) problems that may occur [2]:

- inside or between the elements of the system (intrasystem interference);

- between the elements of the system and the elements of another system that are likely to operate in the same area (inter-system interference);

- between the elements of the system and the electromagnetic environment in which it will operate.

This type of EMI problem is particularly important when multiple systems have to operate simultaneously in a limited space, such as aircraft, freeways and military vessels, and even modern industrial environments [3], [4].

Considering that any winding in which an alternating current flows through it placed into an electromagnetic environmental system creates a leakage magnetic field that could have undesirable effects on the electronic equipment in the respective environment, and can generate inter and intra-system interference.

More recently, the cyber security of both Supervisory control and data acquisition (SCADA) and Industrial
Control Systems (ICS) cannot be ensured if the EMC and EMI aspects listed above are not considered at the first designing step of the life cycles of these systems [5].

Moreover, prolonged exposure of the human body to these low-frequency magnetic fields can have particularly serious health consequences even for low magnetic field intensity values [6].

Thus, some research has shown a high risk of developing leukemia at least for children exposed to fields over $0.4 \mu \mathrm{T}$ [7-11].

\section{PROBLEM FORMULATION}

In this section, a single-phase transformer case it is considered, with the cylinder windings placed onto a single column (Figure 1).

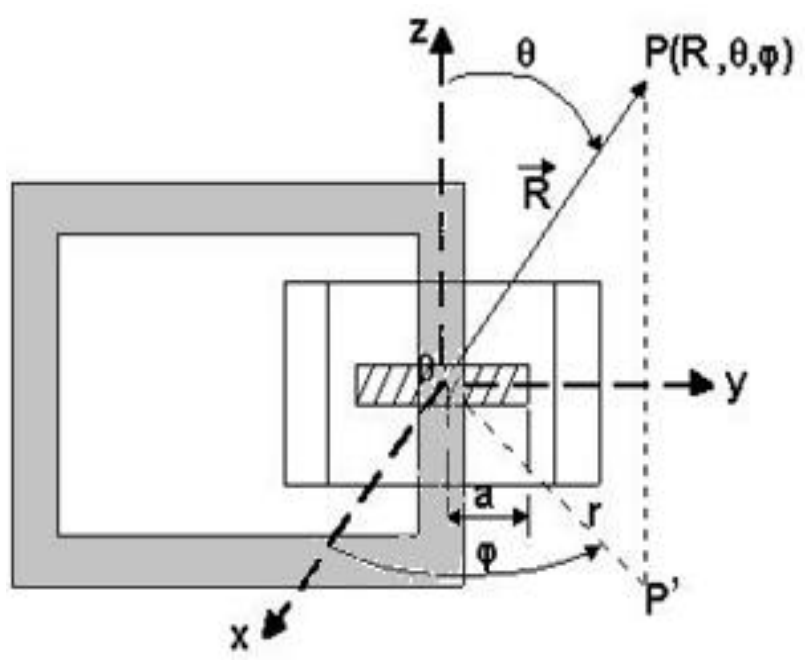

Figure 1. Single-phase transformer with cylinder windings placed onto a single column

In [12] there is presented an algorithm for calculating the intensity of the magnetic field, assimilating the transformer with one equivalent spire having the average radius $a$, disposed in air, having a flow current $I=N_{1} I_{m 10}$, where $N_{l}$ represents the number of turns of the primary winding, and $I_{m l o}$ is the amplitude of the current intensity at idle (no load operation). Thus, the components values of the magnetic field induction at a far distance $\mathrm{R}$ from the center of the equivalent spire (for $R$ $>>$ a) can be calculated based onto the following relations: 


$$
\begin{aligned}
& B_{R} \cong \frac{\mu_{0} I a^{2}}{2 R^{3}} \cos \theta \\
& B_{\theta} \cong \frac{\mu_{0} I a^{2}}{4 R^{3}} \sin \theta
\end{aligned}
$$

The equivalent magnitude of the magnetic field induction can be deduced accordingly:

$$
B(t)=\sqrt{B_{R}^{2}(t)+B_{\theta}^{2}(t)}
$$

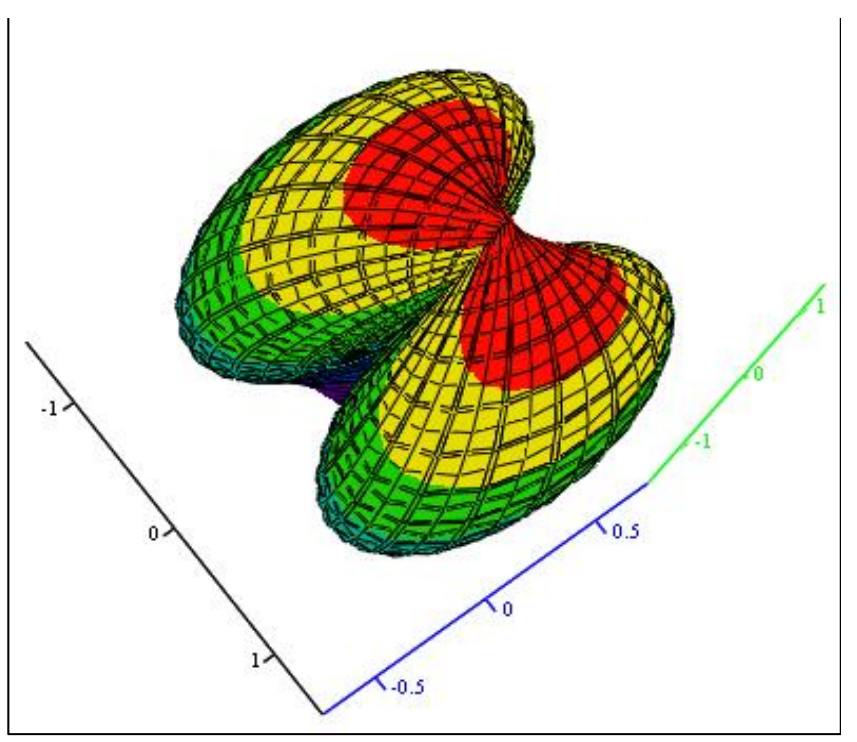

$\mathrm{Br}$

Figure 2. The representation of the induction magnetic field for the component $B_{R}$ obtained based onto relationship (1)

By using analytical equation (1), the representation of the induction magnetic field for the component $B_{R}$ is depicted in the Figure 2.

By using the relationship (2), the other magnetic field induction component, $B \theta$, can be obtained (Figure 3).

On the basis of equation 3 , the representation of the induction magnetic field $B$ is obtained (Figure 4).

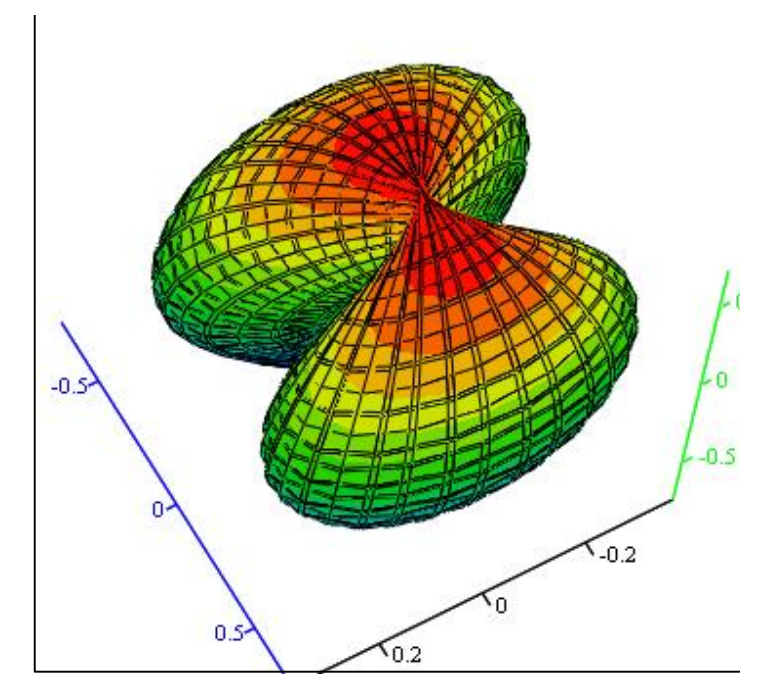

Figure 3. The component $B_{\theta}$ of the magnetic field induction obtained on the basis of relationship (2)

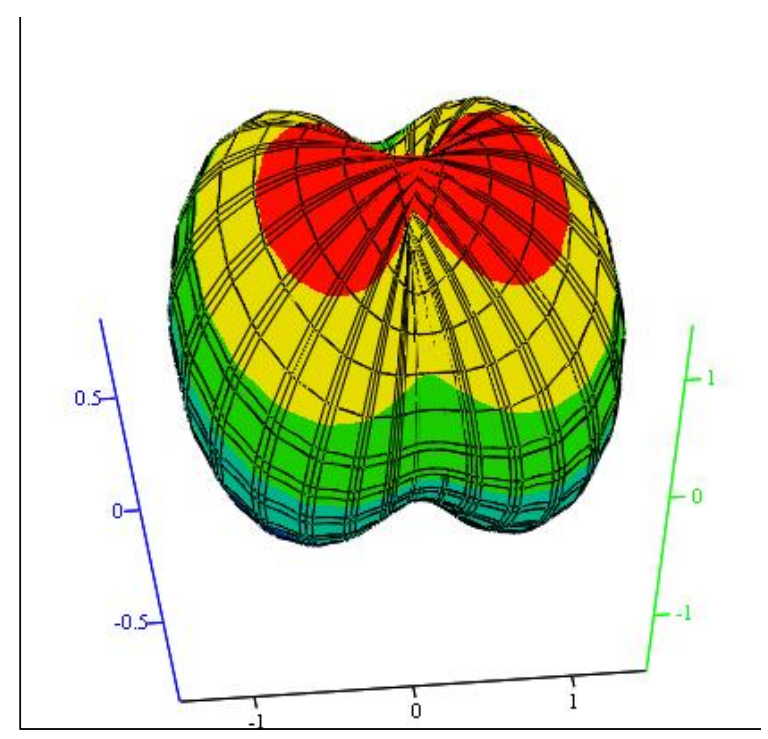

Figure 4. The representation of the induction magnetic field magnitude, $B$, obtained on the basis of relationship (3)

Since the sinusoidal alternative current, of electrical pulsation $\omega$, the final form of the the relations (1), (2), can be obtained:

$$
\begin{aligned}
& B_{R}(t) \cong \frac{\mu_{0} N_{1} I_{m 10} a^{2}}{2 R^{3}} \cos \theta \sin \omega t \\
& B_{\theta}(t) \cong \frac{\mu_{0} N_{1} I_{m 10} a^{2}}{4 R^{3}} \sin \theta \sin \omega t
\end{aligned}
$$

The MathCad implementation of the two above mentioned equations $(4,5)$ are presented adequately in the as follows. 


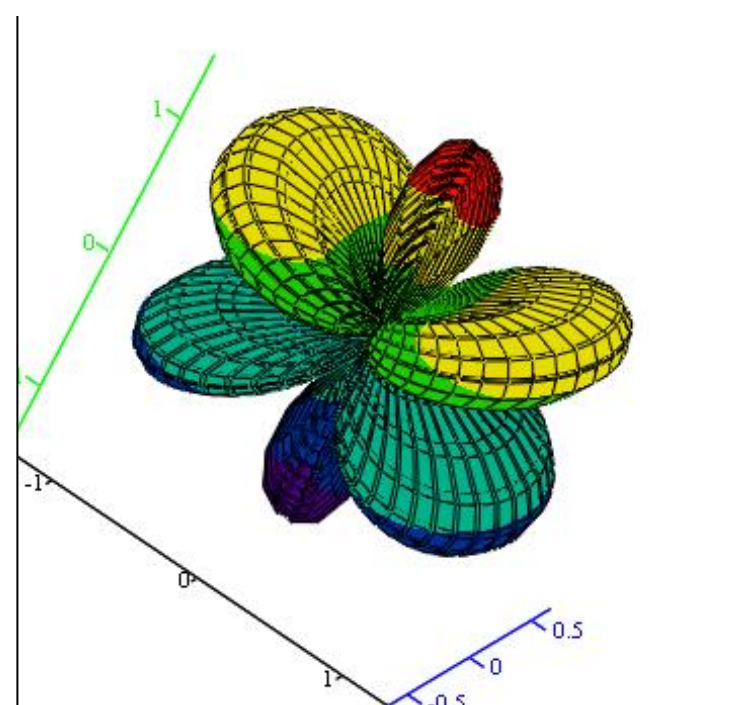

$\mathrm{Br}$

Figure 5. The component $B_{R}$ of the magnetic induction obtained on the basis of relationship (4)

On one hand, the component $B_{R}$ of the magnetic induction has been obtained based on the relationship (4), as in Figure 5. On the other hand, the component $B_{\theta}$ of the magnetic induction (Figure 6) has been obtained on the basis of relationship (5).

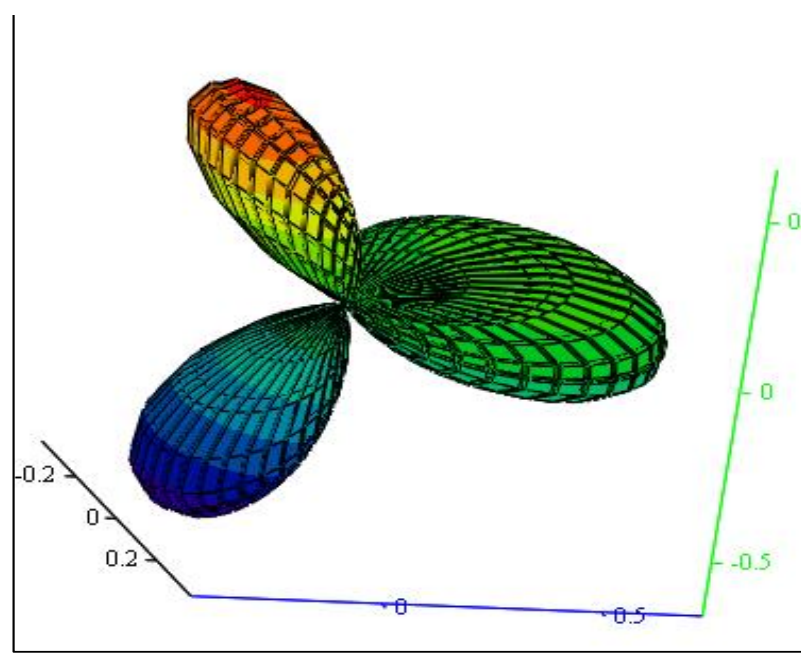

$\mathrm{Bq}$

Figure 6. The component $B_{\theta}$ of the magnetic induction obtained on the basis of relationship (5)

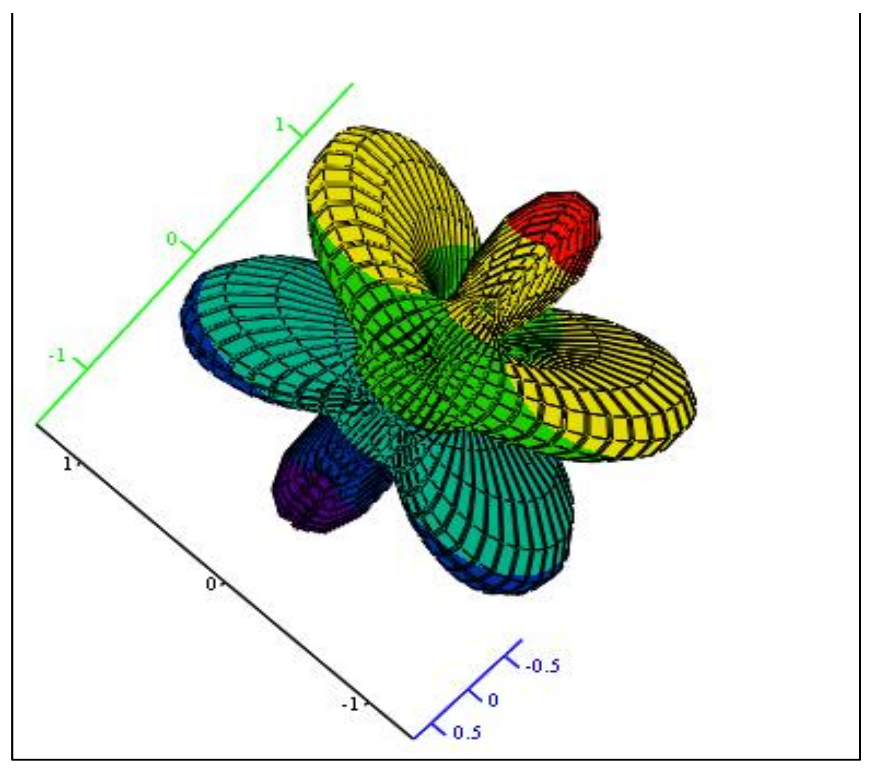

B

Figure 7. The representation of the induction magnetic field magnitude $B(t)$

The representation of the induction magnetic field magnitude $B(t)$ obtained on the basis of relationship (3) with $B_{R}$ and $B_{\theta}$ calculated based on the equations (4) and (5) is presented in the Figure 7.

The studies in the electromagnetic compatibility are analysed in the worst cases possible, consisting of determination of the maximum possible values of the dispersion magnetic field. In this sense, to determine the maximum values of the dispersion induction magnetic field, in case of $R>a$, the following approximation where be taken into account: $\cos \theta=1$. Therefore, the instantaneous values of the expression dispersion induction magnetic field is obtained as:

$$
B(t) \cong B_{R}(t)=\frac{\mu_{0} N_{1} I_{m 10} a^{2}}{2 R^{3}} \sin \omega t
$$

In the Figure 8, Mathcad implementation results of the Equation 6 are presented. 


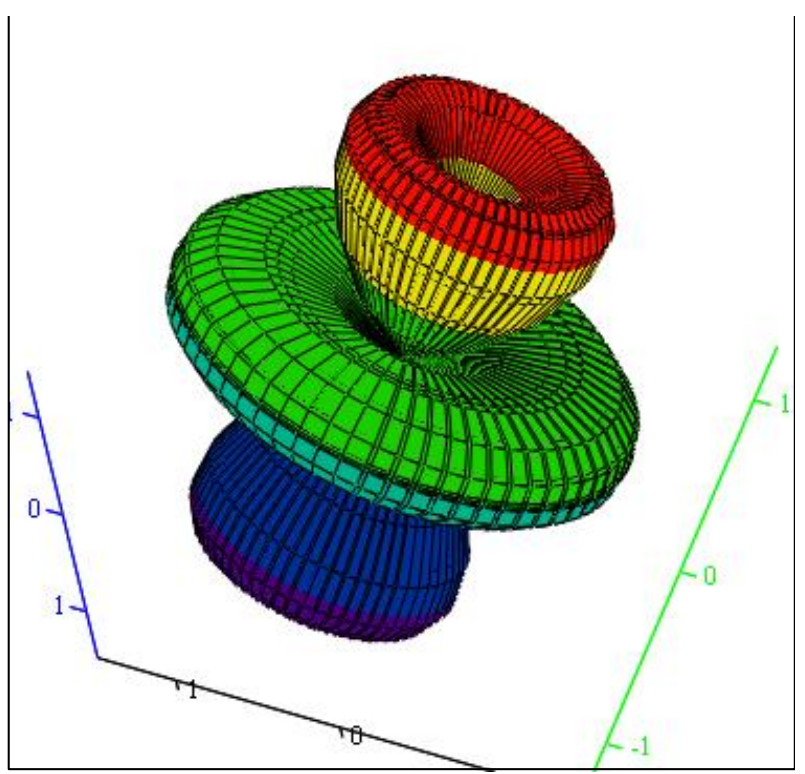

B

Figure 8. Magnetic field induction generated by a single phase transformer, with the cylinder windings arranged on a single column based on the relation (6)

The use of relation (6) can lead to errors in the calculation of the dispersion magnetic induction due to the magnetic permeability $\mu_{0}$, and of the impossibility to establish an equivalent permeability.

At the same time, in practice, the use of the relation (6) is inconvenient because the values for $N_{l}, I_{m 10}$, a are not made available to the user.

Thus, it is preferred to use a relation based on an experimental determination of the perturbed dispersion magnetic field that is obtained starting from the relation (6) [12]. For example, if $B_{0 m}$ is the magnitude of the magnetic induction at the point of observation disposed in the axis of $O z$, at the distance $R_{0}$ from the center of the average coil, the following magnetic induction magnitude on $\mathrm{Oz}$ axis can be deducted:

$$
B_{0 m}=\frac{\sqrt{2} N_{1} I_{10} a^{2} \mu_{0}}{2 R_{0}{ }^{3}}
$$

Any magnetic induction magnitude on $\mathrm{Oz}$ axis at the distance $R>R_{0}, B_{m}$ can be deducted as:

$$
B_{m}=\frac{\sqrt{2} N_{1} I_{10} a^{2} \mu_{0}}{2 R^{3}}
$$

Taken into consideration the above mentioned equations $(7,8)$, the following ratio is obtained

$$
\frac{B_{m}}{B_{0 m}}=\left(\frac{R_{0}}{R}\right)^{3}
$$

Therefore, the magnetic induction magnitude on $\mathrm{Oz}$ axis at the distance $R>R_{0}, B_{m}$, becomes:

$$
B_{m}=B_{0 m}\left(\frac{R_{0}}{R}\right)^{3}
$$

where:

- $B_{0 m}$ is the magnetic induction magnitude at the observation point disposed on the $\mathrm{Oz}$ axis, at the distance $R_{0}$ from the center of the equivalent average coil,

- $B_{m}$ is the magnetic induction magnitude onto $\mathrm{Oz}$ axis, at distance $R>R_{0}$.

Thus, for a given single-phase transformer, if the value $B_{0 m}$ is measured at the observation point disposed on the axis $O z$, at the distance $R_{0}$ from the center of the equivalent coil, the value of the magnetic induction can be calculated at any point on the axis $O z$, with $R>R_{0}$.

The relation (6) can also be used for the purpose of determining the dispersion field developed by a core coil having a single-phase transformer type construction in the mantle. In this case, in relation (6) $I_{m 10}$ represents the alternative component of the flow current intensity through the coil, and $N_{l}$ is the spire number of the coil.

For toroidal ferromagnetic core elements, the magnetic field of dispersion can be determined by the relation:

$$
B=\frac{\mu_{0} I N a_{T}^{2}}{2 R^{3}}
$$

\section{EXPERIMENTAL RESULTS}

The experimental results have been obtained based on a column single-phase transformer, with cylindrical windings placed onto the column, by using the following technical parameters: $S_{n}=2,5 K V A, U_{1 N}=220 \mathrm{~V}$, $U_{2 N}=24 \mathrm{~V}, \quad N_{1}=245, \quad f_{N}=50 \mathrm{~Hz}, N_{2}=32$, $a=0,055 m$. The relation (9) has been used to verify the obtained experimental results. In order to make the specific measurements, the standard apparatus has been used, i.e. a teslameter / magnetometer for alternative magnetic fields of type TA-1.

In this respect, the average value of the magnetic dispersion induction in the $\mathrm{Oz}$ axis was measured (Figure $1)$, for Taking into account the specific parameter of $R_{0}=0,207 \mathrm{~m}$, the following average magnetic induction field has been obtained $B_{0 m e d}=2 \cdot 10^{-4}[T]=200 \mu T$. The following condition is satisfied at the measuring point $R_{0} \gg>a$ Therefore, the values $R_{0}$ and $B_{0 \text { med }}$ are considered as the reference values. Different measurement experiments have been made at different 
points, $R \gg R_{0}$, on the $\mathrm{Oz}$ axis and the differences between the measured average values and those calculated magnetic induction field being between $8 \cdot 10^{-7}$ and $1,3 \cdot 10^{-8}$.

With this respect, at the specific point situated at $R=$ $1,135 \mathrm{~m}$, the following magnetic induction field has been obtained $\quad B_{\text {med measurement }}=0,12 \cdot 10^{-5} \mathrm{~T}=1,2 \mu \mathrm{T}$. Based on the presented theoretical section, by using the equation (9), at the same distance with the previously taken experimental data the following magnetic induction field value has been obtained: $B_{\text {med calculated }}=$ $0,1213258 \cdot 10^{-5} T=1,2132 \mu T$. The obtained errors are under $4 \%$. The higher positive error values have been deducted at the near transformer experimental point. The explanation is due to the additional magnetic induction components from the near field points, which is attenuated with four and five exponent values.

Figures 9 and 10 show the results of the measurements: with the red continuous line the results of the measurements are presented and with the blue dashed line the values of the magnetic induction calculated on the basis of the equation (9). The obtained figures are based on the MATHCAD program. As the interpolation method, the "cspline" function has been used, which performs a cubic interpolation.

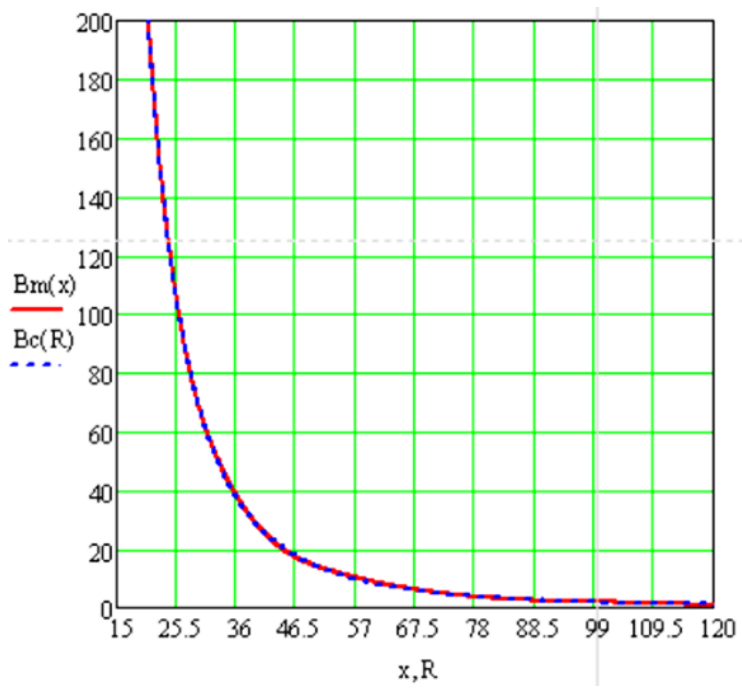

Figure 9. Variation of the magnetic induction field (in $\mu T$ ) as a function of distance (in $\mathrm{cm}$ ) to the source for a single phase transformer with the windings placed onto the column: with blue dotted line - $B c(R)$ - the values calculated based on the equation (9) and with red continuous line -

$B m(x)$ - the magnetic induction values obtained by measurements

Due to the large range of the data values and the very small errors (differences between the calculated and measured values), in Figure 9 the represented variation of the magnetic induction fields, $B m(x)$ and $B c(R)$, are practically identically, the graphs being almost the same. This shows that the relation (9) can be used with very good accuracy for calculating the dispersion induction of the magnetic fields generated by the single-phase transformers with the windings placed onto the column.

In order to show more clearly the error values, the more deeply graphical results has been obtained in the Figure 8: with the red continuous line the one drawn on the measurements and with the blue dashed line the one drawn by performing the calculations using the relation (9). From the Figure 10 it is observed that the differences between the measured values and the calculated ones decrease with the increase of the measurement point distance from the source.

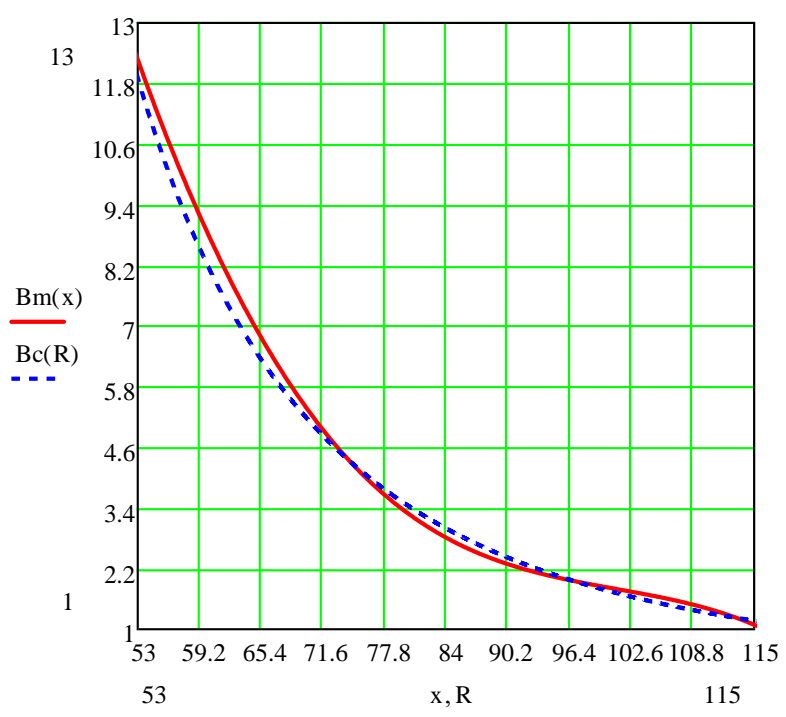

Figure 10. Variation of the magnetic induction field (in $\mu T$ ) as a function of distance (in $\mathrm{cm}$ ) to the source for a single phase transformer with the windings placed onto the column: with blue dotted line - $B c(R)$ - the values calculated based on the equation (9) and with red continuous line $B m(x)$ - the magnetic induction values obtained by measurements

\section{CONCLUSIONS}

Regarding the dispersion induction magnetic field of the single phase transformer the following remarks can be underlined:

- The dispersion induction magnetic field developed by the single phase transformer, at distances $\mathrm{R}$ much larger than the average radius of the equivalent coil, has a magnetic dipole behavior. This aspect is proved by its dependence on the distance from the source with the $1 / R^{3}$ ratio. This dependence indicates the distance from the transformer as the main anti-disturbance measure that can be adopted both for obtaining EMC systems and as a preventive measure for personnel safety.

- Equation (6) can be used in the analysis and prediction of the electromagnetic environment from the definition and design phases of the systems. For practical situations when we do not have all the constructive data of the transformer available, we can use the relation (9).

- From the results of the measurements made onto the single-phase transformer, the relation (9) obtained for the dispersion magnetic induction field, at far distances from 
the transformer, that is to say in distant field $(R>>a)$ leads to corresponding quantifiable values:

Taken into account that the lowest measured value of 1.2 $\mu \mathrm{T}$ was available, (value that differs by $1.09 \%$ from that calculated one) and comparing this value with the results of studies from [6-11] (i.e.0.4 $\mu \mathrm{T}$ ), it turns out that all the measured values can be potentially dangerous for the human body in case of prolonged exposure.

\section{REFERENCES}

[1] Schwab, A. Compatibilitatea electromagnetică, Bucureşti: Editura Tehnică, 1996.

[2] Duff, William G. Handbook Series on Electromagnetic Interference and Compatibility Vol. 7, Gainesville, Virginia, USA : Interference Control Technologies, Inc. Gainesville, Virginia, 1988.

[3] Vasile Solcanu, Marian Gaiceanu, The method of compensating non -linear effects due to disturbing signals in the military operations theatres, Analele Universitatii "Eftimie Murgu", Fascicula de Inginerie, 2019, Vol. Vol. 26, No.1,pag. 213-220, ISSN 1453 - 7397.

[4] Vasile Solcanu, Marian Gaiceanu, Alexandru Sotir, Gheorghe Samoilescu, Mircea Constantinescu, The results of the electromagnetic field measurements performed on a military maritime ship to determine the effectiveness of a radio-absorbent material, Galati : 6th International Symposium on Electrical and Electronics Engineering (ISEEE), 2019.

[5] SANS Industrial Control Systems, https://ics.sans.org/ (accessed 27.03.2020).

[6] Yvan Touitou, Brahim Selmaoui, The effects of extremely low-frequency magnetic fields on melatonin and cortisol, two marker rhythms of the circadian system, Dialogues Clin Neurosci, 14(4): 381-399, https://www.ncbi.nlm.nih.gov/pmc/articles/PMC35 53569/, 2012 Dec.

[7] Dangerous magnetic field exposure near transformer substation in the building s.l.: https://electricalengineering-portal.com/magnetic-field-exposuretransformer-substation, 2017.

[8] G. Rosu, G. Samoilescu, M.C. Rau, O. Baltag, Aspects regarding the occupational and nonoccupational exposure to low frequency and radiofrequency electromagnetic fields, Craiova: 2016 International Conference on Applied and Theoretical Electricity (ICATE), pp.1-6, doi: 10.1109/ICATE.2016.7754700, 2016.

[9] Vasile Solcanu, Marian Gaiceanu, Alexandru Sotir, Gheorghe Samoilescu, Mircea Constantinescu, The results of the electromagnetic field measurements performed on a military maritime ship to determine the effectiveness of a radio-absorbent material, Galati : 6th International Symposium on Electrical and Electronics Engineering (ISEEE), 2019.

[10] Pavel, Ing. Ionel. Cercetari privind supravegherea campurilor maganetice generate de sistemele de alimentare cu energie electrica -Rezumatul Tezei de doctorat - Iasi: Universitatea Tehnica "Ghe. Asachi", 2019.

[11] Ahlbom A., Day N., Feychting M., et al. A pooled analysis of magnetic fields and childhood leukemia. Br J Cancer. 2000;83:692-698.

[12] Rapoarte de cercetare in cadrul Programului Naţional CEEX 2005: "Ecologie Electromagnetica caracterizarea surselor, diagnosticarea efectelor, prevenirea si combaterea lor”, 2005-2008. 